\title{
Phase diagram of quantized vortices in a trapped Bose-Einstein condensed gas
}

\author{
S. Stringari \\ Dipartimento di Fisica, Università di Trento, \\ and Istituto Nazionale di Fisica della Materia, I-3850 Povo, Italy
}

(19 December 1998)

We investigate the thermodynamic stability of quantized vortices in a dilute Bose gas confined by a rotating harmonic trap at finite temperature. Interatomic forces play a crucial role in characterizing the resulting phase diagram, especially in the large $N$ Thomas-Fermi regime. We show that the critical temperature for the creation of stable vortices exhibits a maximum as a function of the frequency of the rotating trap and that the corresponding transition is associated with a discontinuity in the number of atoms in the condensate. Possible strategies for approaching the vortical region are discussed.

PACS numbers: 03.75.F, 05.30.J, 32.80 Pj, 67.40.Db

The occurrence of quantized vortices in superfluids has been the object in the past of fundamental theoretical and experimental work 1 [ 5]. After the experimental realization of Bose-Einstein condensation in trapped alkali gases [6], the structure of vortices in these novel manybody sytems has soon attracted the attention of theorists [79] (for a recent review on various theoretical aspects of Bose-Einstein condensation in trapped gases, see [10]). Special attention has been devoted, in particular, to the calculation of the critical frequency needed to create a stable vortex in the rotating frame. This quantity is of crucial importance in view of the experimental possibility of creating vortices by rotation of the confining trap. In systems interacting with repulsive forces the critical frequency turns out to be smaller than the oscillator frequency characterizing the confining harmonic potential and to decrease smoothly with the number of atoms in the condensate. The purpose of this work is to discuss the behavior of the critical frequency at finite temperature, pointing out the crucial role played by the interactions, especially in the large N, Thomas-Fermi regime, where the system behaves like a Bogoliubov gas. We will show that, due to the harmonic nature of the confinement, the phase diagram for the vortical configurations of these dilute Bose gases exhibits new interesting features which are absent in dense superfluids.

Let us consider a trap rotating with frequency $\omega$ along the $z$-axis. The equilibrium configuration of the system in the rotating frame is obtained by minimizing the free energy $F=E-T S$, using the Hamiltonian $H(\omega)=H-\omega J_{z}$ where $H$ and $J_{z}$ are the Hamiltonian and the angular momentum (third component) of the many-body system, evaluated in the laboratory frame. A vortical configuration is thermodynamically stable if the trap rotates with frequency $\omega$ larger than the critical frequency

$$
\omega_{c}=\frac{F_{v}-F_{0}}{\left\langle J_{z}\right\rangle_{v}-\left\langle J_{z}\right\rangle_{0}},
$$

fixed by the difference between the free energy of the configurations with and without the vortex and by the corresponding difference of the angular momentum. The stability criteria for vortices, including the possible occurrence of metastable configurations, have been the object of several recent theoretical studies [11 14]. In particular one has to distinguish between configurations where the system is stable against small deformations of the system (local stability) and configurations where the system is in full thermodynamic equilibrium (global stability). For frequencies smaller than the critical frequency (1) the system, in the presence of a vortex, is not thermodynamically stable, but can be locally stable (metastability) [11.12]. For even smaller frequencies the vortex is unstable also locally and exhibits elementary excitations with negative energies [13,15], unless one considers toroidal geometries. In the following we will discuss only configurations in which the system is in full thermal equilibrium in the rotating frame.

We will consider a gas confined by an axially symmetric harmonic potential of the form $V_{e x t}=m\left(\omega_{z}^{2} z^{2}+\omega_{\perp}^{2} r_{\perp}^{2}\right) / 2$ with $r_{\perp}^{2}=x^{2}+y^{2}$. Actually, in order to achieve thermalization in the rotating frame the trap should contain an asymmetry in the $x y$-plane. One however expects that, unless the gas is too cold, even very small asymmetries are sufficient to ensure fast thermalization, so that one can safely use symmetric potentials for the calculation of the vortical configuration as well as of the relevant thermodynamic functions and, at the same time, assume thermal equilibrium in the rotating frame.

In the absence of two-body interactions the critical frequency $\omega_{c}$ is easily calculated and coincides with the radial oscillator frequency $\omega_{\perp}$. In interacting gases the value of $\omega_{c}$ can be calculated, at zero temperature, by solving the Gross-Pitaevskii equation for the order parameter 8]. In this case equation (3) takes the simpler form $\omega_{c}=\left(E_{v}-E_{0}\right) / N \hbar$ where $E_{v}$ and $E_{0}$ are the energies of the ground state configurations with and without the vortex, respectively, and we have considered a vortex with one quantum of circulation: $\left\langle J_{z}\right\rangle_{v}=N \hbar$, $\left\langle J_{z}\right\rangle_{0}=0$. Typical values of $\omega_{c}$ correpond to a few tens of Hertz. Analogously, for each value of $\omega$, one can calculate a critical value $N_{0}^{c}$ such that, for $N_{0} \geq N_{0}^{c}$ the 
vortex corresponds to a stable configuration in the frame rotating with frequency $\omega$. Actually the Gross-Pitaevskii equation exhibits a scaling behaviour in the interaction parameter $N_{0} a / a_{h o}$, where $a$ is the $s$-wave scattering length, which will be always assumed to be positive, and $a_{h o}=\sqrt{\hbar / m \omega_{h o}}$ is the oscillator length relative to the geometrical average $\omega_{h o}=\left(\omega_{z} \omega_{\perp}^{2}\right)^{1 / 3}=\lambda^{1 / 3} \omega_{\perp}$ where $\lambda=\omega_{z} / \omega_{\perp}$ is the deformation parameter of the trap. As a consequence of scaling one can always write the critical number $N_{0}^{c}$ in the form

$$
N_{0}^{c}(\omega) \frac{a}{a_{h o}}=f_{\lambda}\left(\frac{\omega}{\omega_{\perp}}\right)
$$

where $f_{\lambda}$ is a function that can be calculated by solving the Gross-Pitaevskii equation for each value of $\lambda$. When the dimensionless parameter $N_{0} a / a_{h o}$ is much larger than 1 (Thomas-Fermi limit) the solution of the Gross-Pitaevskii equation gives the following result 16 (see also [7, 17]) for the critical frequency

$$
\frac{\omega_{c}}{\omega_{\perp}}=\frac{5 \lambda^{1 / 3} a_{h o}^{2}}{2 R_{\perp}^{2}} \ln \frac{0.67 R_{\perp}}{\xi},
$$

where $R_{\perp}=\lambda^{1 / 3} a_{h o}\left(15 N_{0} a / a_{h o}\right)^{1 / 5}$ is the ThomasFermi radius of the condensate in the $x y$-plane, while $\xi=a_{h o}\left(15 N_{0} a / a_{h o}\right)^{-1 / 5}$ is the healing length, which fixes the size of the vortex core. The Thomas-Fermi result (3) allows one to determine in a simple way the function $f_{\lambda}$ which turns out to scale as $\lambda^{-5 / 6}$. In the opposite limit, when the parameter $N_{0} a / a_{h o}$ is very small, one can use a perturbative treatment of the interaction yielding the result $\omega_{c} / \omega_{\perp}=1-\lambda^{1 / 3}(8 \pi)^{-1 / 2} N_{0} a / a_{h o}$. The quasi ideal gas limit is not however relevant for the available experimental configurations since $N_{0} a / a_{h o}$ is always much larger than 1 . In fig. 1 we compare the results for the function $\lambda^{5 / 6} f_{\lambda}$, calculated by solving the GrossPitaevskii equation with the prediction of the ThomasFermi approximation for two different values of the deformation parameter $(\lambda=\sqrt{8}$ and $\lambda=1)$. One sees that the Thomas-Fermi limit is rather accurate for $\omega \leq 0.5 \omega_{\perp}$.

From an experimental point of view the creation of a vortex by adiabatic increase of the rotational frequency of the trap up to $\omega_{c}$ might not be the safest procedure at very low temperature. In fact one expects that in this case the system will exhibit a high barrier for the nucleation of vortices as happens in superfluid helium [5]. Though the nucleation process in dilute gases might behave differently from the one of a dense supefluid, one nevertheless expects that a safer procedure to generate vortices is first to rotate the gas at higher temperature (in case, also above the critical temperature for BoseEinstein condensation) and then to cool it via evaporation. This strategy has motivated the present investigation of the phase diagram of vortices at finite temperature.
Let us first discuss the behaviour of the critical temperature $T_{c}$ as a function of the rotational frequency. We will suppose the system to be in thermal equilibrium in the frame rotating with frequency $\omega$. A first estimate of the critical temperature is obtained by taking the ideal gas model. In the absence of rotations and for large values of $N$ this model predicts the result $T_{c}^{0}=0.94\left(\hbar \omega_{h o} / k_{B}\right) N^{1 / 3}$.

The effect of the rotation is to change the shape of the distribution function of the thermal atoms so that the number of atoms out of the condensate takes the form

$$
\begin{aligned}
N_{T} & =\frac{1}{(2 \pi \hbar)^{3}} \int d \mathbf{r} d \mathbf{p}\left\{\operatorname { e x p } \left[\frac{p^{2}}{2 m}+\frac{m}{2}\left(\omega_{z}^{2} z^{2}+\omega_{\perp}^{2} r_{\perp}^{2}\right)\right.\right. \\
& \left.\left.-\omega\left(x p_{y}-y p_{x}\right)\right] / k_{B} T-1\right\}^{-1} .
\end{aligned}
$$

Result (4) holds for $T \leq T_{c}$ where the chemical potential of the non interacting model vanishes in the thermodynamic limit. Notice that, unless a vortex is formed, the rotation has instead no effect on the condensate, whose wave function has zero angular momentum since the confining trap is axially symmetric. By using the transformation $\mathbf{p} \rightarrow \mathbf{p}-m \omega \times \mathbf{r}$ the integral (4) takes the same form as in the absence of rotation, with an effective radial frequency $\omega_{\perp}(\omega)=\left(\omega_{\perp}^{2}-\omega^{2}\right)^{1 / 2}$. This reflects the role of the centrifugal force which is consequently responsible for a lowering of the critical temperature $T_{c}$ according to

$$
T_{c}(\omega)=T_{c}^{0}\left(1-\frac{\omega^{2}}{\omega_{\perp}^{2}}\right)^{1 / 3}
$$

where $T_{c}^{0}$ is the critical temperature in the absence of rotation. In the same model the temperature dependence of the condensate fraction is given by:

$$
\frac{N_{0}}{N}=1-\left(\frac{T}{T_{c}(\omega)}\right)^{3} .
$$

The lowering of $T_{c}$ is dramatic near $\omega_{\perp}$ where the system becomes unstable. In this case the centrifugal force exactly cancels the confining effect of the harmonic potential. For confining potentials growing faster than $r^{2}$ at large distances the critical temperature would instead differ from zero for any value of the rotating frequency. The behaviour exhibited by dilute gases in harmonic traps should be compared with the one of dense liquids, like superfluid helium, where the critical temperature is practically unaffected by the rotation.

The next step is to assume that the $T=0$ relationship $N_{0}^{c}(\omega)$ of eq.(2) between the frequency $\omega$ and the minimum number of atoms in the condensate required to have a stable vortex, is not changed at finite temperature. This relationship allows one to calculate, through eq.(6), the critical temperature $T_{v}$, below which the vortex corresponds to a stable configuration in the frame rotating with frequency $\omega$. We find the useful result 


$$
T_{v}(\omega)=T_{c}^{0}\left(1-\frac{\omega^{2}}{\omega_{\perp}^{2}}\right)^{1 / 3}\left(1-\frac{a_{h o}}{a N} f_{\lambda}\left(\frac{\omega}{\omega_{\perp}}\right)\right)^{1 / 3}
$$

where we have explicitly used eq.(5) for the critical temperature. Equation (77) points out the crucial role played by two-body interactions. In particular, by increasing the value of the dimensionless parameter $N a / a_{h o}$, the curve $T_{v}$ is pushed to the left, making the region of stability for vortices wider and wider. In fig.2 we show the critical curves $T_{c}(\omega)$ (dashed line) and $T_{v}(\omega)$ (full line). The function $f_{\lambda}$ entering eq.(7) was obtained by solving the Gross-Pitaevskii equation. For temperatures below $T_{c}(\omega)$ the gas exhibits Bose-Einstein condensation. However only for temperatures below $T_{v}(\omega)$ will the vortex correspond to the equilibrium configuration of the system.

In deriving the main result (7) we have made several approximations that it is worth discussing explicitly. These concern the use of the ideal gas predictions (5) and (6) for the critical temperature and the thermal depletion of the condensate, as well as the use, at finite temperature, of the $T=0$ relationship between the rotational frequency and the critical value of $N_{0}^{c}$, given by the solution of the Gross-Pitaevskii equation.

Neglecting interaction effects in the evaluation of the critical temperature is a very good approximation since near $T_{c}$ the gas is extremely dilute. Indeed self-consistent mean field [18,10] as well as ab initio [19] calculations show that $T_{c}$ is lowered by interactions only by a few percent. The effects on the thermal depletion of the condensate are instead expected to be more important because of the sizable renormalization of the chemical potential due to the presence of the condensate. For the same reason one expects that the relationship (2) between the frequency and the critical value of $N_{0}^{c}$ should exhibit a temperature dependence.

In order to understand the importance of the above effects let us calculate the chemical potential $\mu=\partial F / \partial N$ of the gas with and without the vortex. Also at finite temperature the chemical potential can be calculated, with good approximation, by solving the GrossPitaevskii equation for the condensate with a fixed value of $N_{0}$. In the absence of vortices and for large values of $N_{0} a / a_{h o}$ one can use the Thomas-Fermi formula $\mu_{0}\left(N_{0}\right)=\frac{1}{2} \hbar \omega_{h o}\left(15 N_{0} a / a_{h o}\right)^{2 / 5}$. If a vortex is present the chemical potential will contain an extra term $\delta \mu=$ $\frac{3}{5} \hbar \omega_{c}$ with $\omega_{c}$ given by eq.(3). Notice that both $\mu_{0}$ and $\delta \mu$ are fixed by the value of $N_{0}$. However, for a given value of $N$ and $T$, the value of $N_{0}$ differs depending on whether the vortex is present or not. This effect is absent if one uses the ideal gas prediction (6). By treating the interaction effect as a small perturbation one finds the result 10

$$
\frac{N_{0}}{N}=1-\frac{T^{3}}{T_{c}^{3}(\omega)}-\frac{\zeta(2)}{\zeta(3)} \frac{\mu T^{2}}{k_{B} T_{c}^{3}(\omega)} .
$$

which generalizes the ideal gas prediction (6). The above equation shows that, because of interactions, the number of atoms in the condensate with and without the vortex differ, to the lowest order in the interaction, by the amount

$$
\frac{\delta N_{0}}{N}=-\frac{\zeta(2)}{\zeta(3)} \frac{\delta \mu T^{2}}{k_{B} T_{c}^{3}(\omega)} .
$$

The discontinuity $\delta N_{0}$ in the number of atoms in the condensate is not very large (at the maximum of the curve $T_{v}$ of fig.2 one finds $\delta N_{0} \sim 200$ ), but should be taken into account for a safe estimate of the critical frequency. In fact the relevant change in the chemical potential is given by $\mu_{v}(N, T)-\mu_{0}(N, T)=\left(\partial \mu_{0} / \partial N_{0}\right) \delta N_{0}+\delta \mu$. By using (8) and (9) one finds that the leading corrections, due to the interaction term in (8), cancel out. This suggests that, at finite temperature, the critical frequency can be safely calculated using the $T=0$ solution of the Gross-Pitaevskii equation and the ideal gas expression (6) for $N_{0}$. In conclusion the main result (7) for the critical temperature for vortices is expected to represent a rather good estimate also when $T_{v}$ is comparable to $T_{c}$. Notice that the value of $N_{0}$ on the line $T_{v}$ can be nevertheless significantly smaller than the ideal gas prediction (6) because of the interaction term in (8). This effect should lower the height of the barrier at the transition and hence favour the nucleation of the vortex.

A possible way to approach to the vortical region is through an adiabatic transformation. Suppose that, starting from a system initially at rest in the laboratory, we switch on the rotation of the trap in an adiabatic way. Since the entropy of the gas is given, in first approximation, by the ideal gas value $S=$ $4 k_{B} N(\zeta(4) / \zeta(3))\left(T / T_{c}(\omega)\right)^{3}$ the adiabatic increase of the frequency has the effect of lowering the temperature. If the initial temperature is below $T_{c}$ the transformation will eventually bring the system into the vortical region. Notice that this scenario does not require that the initial temperature be much smaller than $T_{c}$. This is important for at least two reasons: first the presence of a large fraction of thermal atoms is crucial to favour the thermalization of the gas in the rotating frame; second, the nucleation of the vortex may become easier if the number of atoms in the condensate is not too large.

Once the vortex is created a major problem is its experimental detection. Recently, various methods have been proposed to detect vortices in these trapped gases. They include the imaging of the vortex core following the expansion of the atomic cloud [20], the occurrence of frequency shifts in the collective oscillations 117,21 and the occurrence of dislocations in the interference patterns between condensates containing vortical configurations [22].

In the most interesting vortical region the system is expected to exhibit further interesting features. In fact increasing the rotational frequency will favour the creation of more complex vortical configurations, associated with 
the occurrence of 2 or more vortices 23,24. In principle vortices with higher quanta of circulation are also possible. However these configurations are likely unstable [5,14. A final interesting question concerns the stability of elementary excitations in the rotating gas. In 25] it has been shown that, for large frequencies, the surface excitations of the condensate become unstable. This happens for frequencies larger, but not tremendously larger, than the critical frequency needed to create a vortex. For example with $N=10^{4}$ atoms in a symmetric trap at $T=0$ it is found [25] that for the critical frequency for generating a surface instability is $\omega / \omega_{\perp} \sim 0.5$, while the one for the vortex instability is $\sim 0.35$. The influence of the vortex on the stability of such excitations [12] as well as the possible occurrence of new scenarios associated with the "condensation" of surface excitations are challenging questions for future investigation.

I would like to thank F. Dalfovo, J. Dalibard, S. Giorgini and L. Pitaevskii for many useful discussions. This project was supported by the Istituto Nazionale per la Fisica della Materia through the Advanced Research Project on BEC.

Figure Captions.

Fig.1. Function $\lambda^{5 / 6} f_{\lambda}$ calculated for two different values of $\lambda$ (open circles: $\lambda=1$; solid circles: $\lambda=\sqrt{8}$ ). The prediction obtained from eq. (3) is also reported (dashed line). Frequencies are in units of $\omega_{\perp}$.

Fig.2. Phase diagram for vortices in a harmonically trapped Bose gas $\left(N=10^{4}, a / a_{h o}=7.36 \times 10^{-3}\right.$ and $\lambda=1)$. Frequencies are in units of $\omega_{\perp}$.

[1] L. Onsager, Nuovo Cimento, 6, 249 (1949).

[2] R.F. Feynman, in Progress in Low Temperature Physics, vol. I, ed. C.J. Gorter (North Holland, 1955)

[3] W.F. Vinen, Proc. Roy. Soc. A 260, 218 (1961)

[4] E.J. Yarmchuck, M.J.V. Gordon, and R.E. Packard, Phys. Rev. Lett. 43, 214 (1979)

[5] R. Donnelly, Quantized Vortices in Helium II (Cambridge University Press, U.K. 1991).

[6] M.H. Anderson et al., Science, 269, 198 (1995); K.B. Davis et al. Phys. Rev. Lett. 75, 3969 (1995); C.C. Bradley et al., Phys. Rev. Lett. 78, 985 (1997) (see also Phys. Rev. Lett. 75, 1687 (1995).

[7] G. Baym and C. Pethick, Phys. Rev. Lett. 76, 6 (1996).

[8] F. Dalfovo and S. Stringari, Phys. Rev. A 53, 2477 (1996).

[9] M. Edwards et al., Phys Rev. A 53, R1950 (1996).

[10] F. Dalfovo, L. Pitaevskii, S. Giorgini and S. Stringari, cond-mat/9806038, Rev. Mod. Phys., April 1999.

[11] A.L. Fetter, J. Low Temp. Phys. 113, 189 (1998); A.A. Svidzinsky and A.L. Fetter, cond-mat/9811348.

[12] T. Isoshima and K Machida, cond-mat/9807250 and cond-mat/9811367.
13] D.S. Rokhsar, Phys. Rev. Lett, 79, 2164 (1997); condmat/9709212.

[14] H. Pu, C.K. Law, J.H. Eberly, and N.P. Bigelow, condmat/9807362.

[15] R.J. Dodd et al. Phys. Rev. A 56, 587 (1997).

[16] E. Lundh, C.J. Pethick, and H. Smith, Phys. Rev. A 55, 2126 (1997).

[17] S. Sinha, Phys. Rev. A 55, 4325 (1997).

[18] S. Giorgini, L. Pitaevskii, and S. Stringari, Phys. Rev. A 54, 4633 (1996); J. Low Temp. Phys. 109, 309 (1997).

[19] W. Krauth Phys. Rev. Lett. 77, 3695 (1996).

$[20]$ E. Lundh, C.J. Pethick, and H. Smith, condmat/9805351.

21 F. Zambelli and S. Stringari, Phys. Rev. Lett. 81, 1754 (1998); A.A. Svidzinsky and A.L. Fetter, Phys. Rev. A, 58, 3168 (1998).

[22] E.L. Bolda and D.F. Walls, cond-mat/9807345 J. Tempere and J.T. Devreese, Sol. St. Comm., in press.

[23] D.A. Butts and D.S. Rokhsar, Nature, in press.

[24] Y. Castin and R. Dum, to be published.

[25] F. Dalfovo et al., Phys. Rev. A 56, 3840 (1997). 


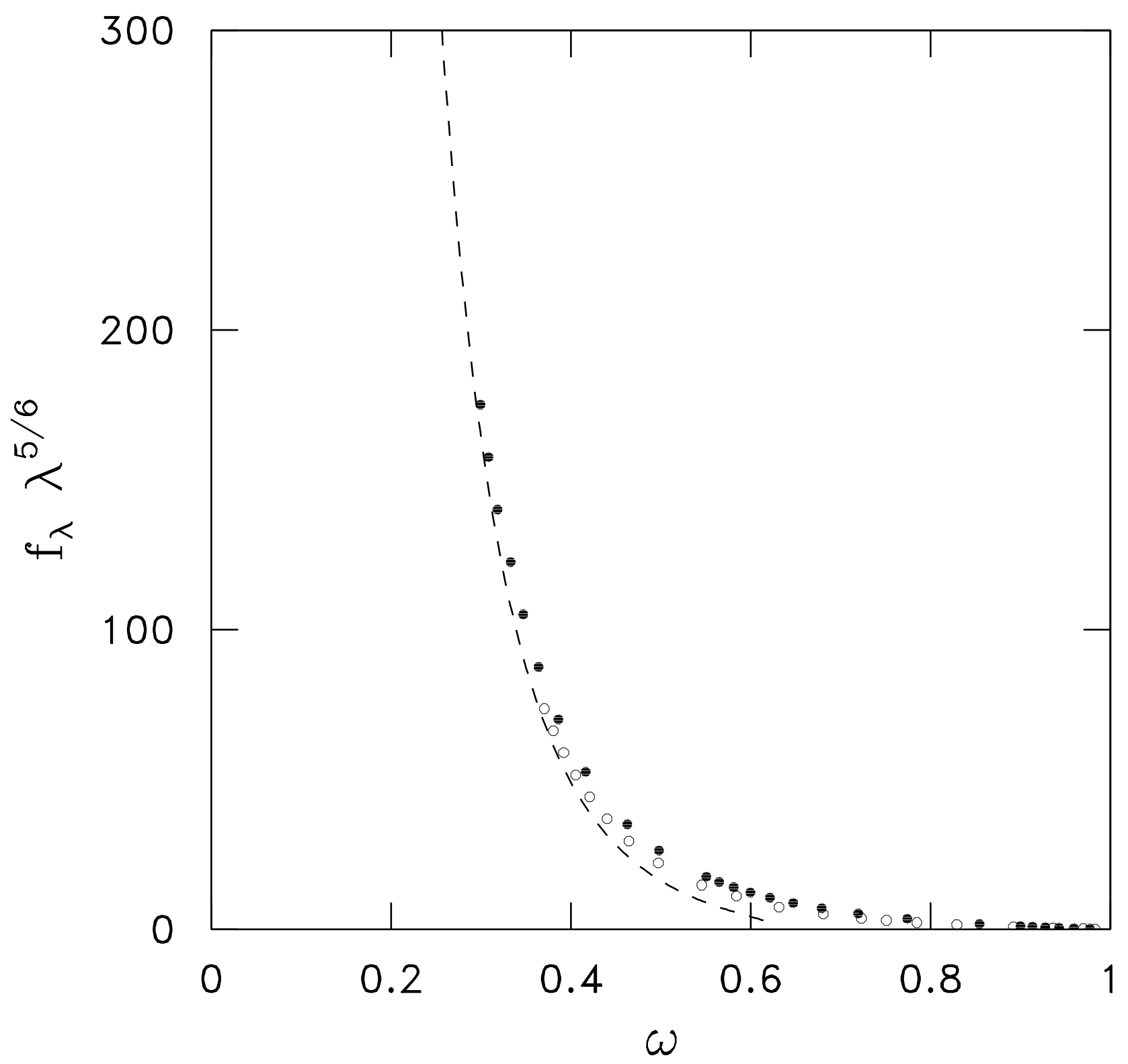




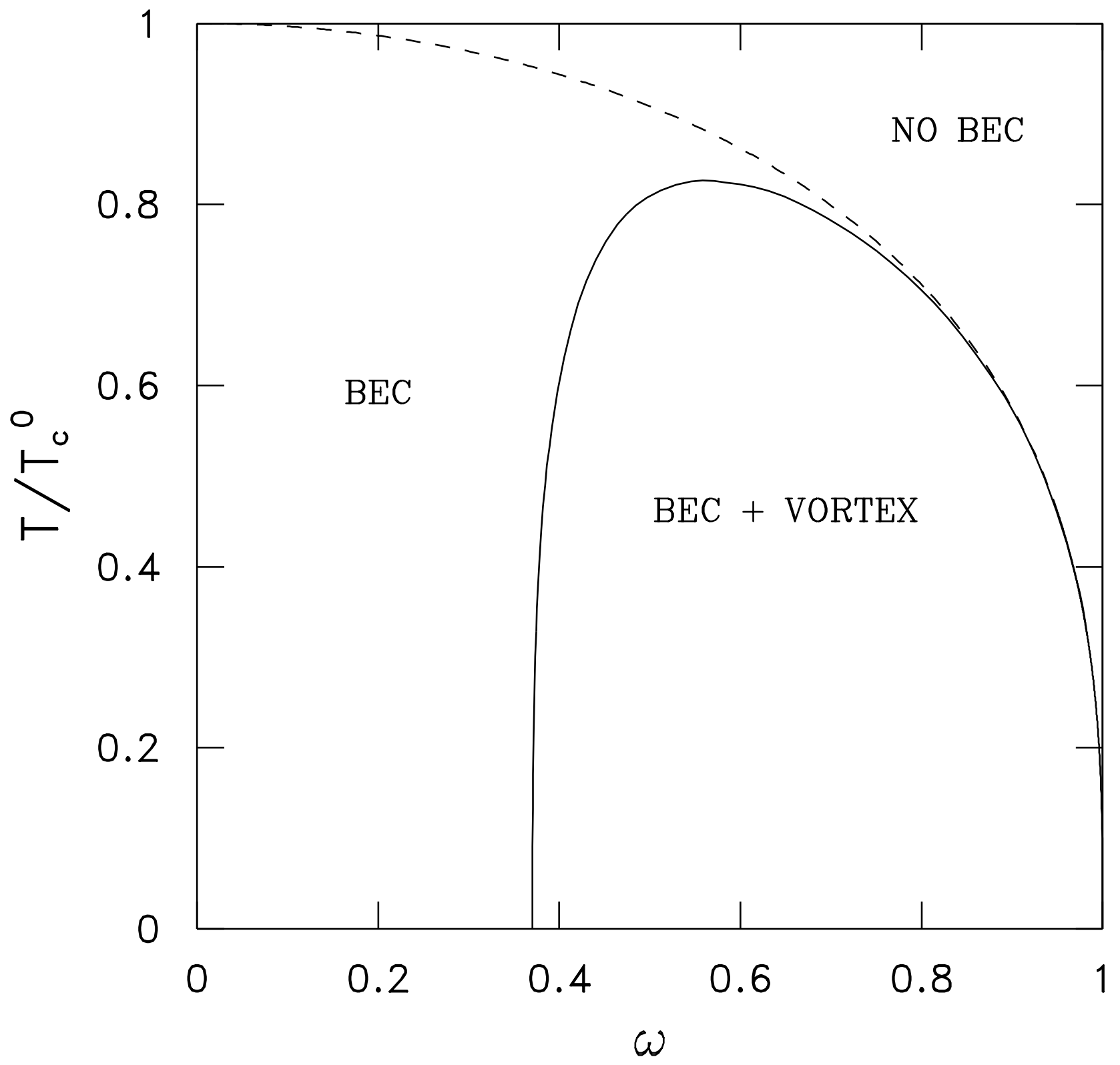

